\title{
Some Properties of the Sequence Space $E_{n}^{q}(\mathscr{M}, p, s,\|\cdot, \ldots, \cdot\|)$
}

\author{
Kuldip Raj and Sunil K. Sharma \\ School of Mathematics, Shri Mata Vaishno Devi University, Katra, Jammu and Kashmir 182 320, India \\ Correspondence should be addressed to Kuldip Raj; kuldeepraj68@rediffmail.com
}

Received 29 March 2013; Revised 6 June 2013; Accepted 6 June 2013

Academic Editor: Palle E. Jorgensen

Copyright (C) 2013 K. Raj and S. K. Sharma. This is an open access article distributed under the Creative Commons Attribution License, which permits unrestricted use, distribution, and reproduction in any medium, provided the original work is properly cited.

We introduce the sequence space $E_{n}^{q}(\mathscr{M}, p, s,\|\cdot, \ldots, \cdot\|)$ defined by a Musielak-Orlicz function $\mathscr{M}=\left(M_{k}\right)$. We also study some topological properties and prove some inclusion relations involving this space.

\section{Introduction and Preliminaries}

The concept of 2-normed spaces was initially developed by Gähler [1] in the mid-1960s, while one can see that of $n$-normed spaces in Misiak [2]. Since then, many others have studied this concept and obtained various results; see Gunawan [3,4] and Gunawan and Mashadi [5]. Let $n \in \mathbb{N}$ and $X$ be a linear space over the field $\mathbb{K}$, where $\mathbb{K}$ is the field of real or complex numbers of dimension $d$, where $d \geq n \geq 2$. A real valued function $\|\cdot, \ldots, \cdot\|$ on $X^{n}$ satisfying the following four conditions:

(1) $\left\|x_{1}, x_{2}, \ldots, x_{n}\right\|=0$ if and only if $x_{1}, x_{2}, \ldots, x_{n}$ are linearly dependent in $X$;

(2) $\left\|x_{1}, x_{2}, \ldots, x_{n}\right\|$ is invariant under permutation;

(3) $\left\|\alpha x_{1}, x_{2}, \ldots, x_{n}\right\|=|\alpha|\left\|x_{1}, x_{2}, \ldots, x_{n}\right\|$ for any $\alpha \in \mathbb{K}$; and

(4) $\left\|x+x^{\prime}, x_{2}, \ldots, x_{n}\right\| \leq\left\|x, x_{2}, \ldots, x_{n}\right\|+\left\|x^{\prime}, x_{2}, \ldots, x_{n}\right\|$

is called an $n$-norm on $X$, and the pair $(X,\|\cdot, \ldots, \cdot\|)$ is called an $n$-normed space over the field $\mathbb{K}$.

For example, we may take $X=\mathbb{R}^{n}$ being equipped with the $n$-norm $\left\|x_{1}, x_{2}, \ldots, x_{n}\right\|_{E}=$ the volume of the $n$-dimensional parallelopiped spanned by the vectors $x_{1}, x_{2}, \ldots, x_{n}$ which may be given explicitly by the formula

$$
\left\|x_{1}, x_{2}, \ldots, x_{n}\right\|_{E}=\left|\operatorname{det}\left(x_{i j}\right)\right| \text {, }
$$

where $x_{i}=\left(x_{i 1}, x_{i 2}, \ldots, x_{i n}\right) \in \mathbb{R}^{n}$ for each $i=1,2, \ldots, n$. Let $(X,\|\cdot, \ldots, \cdot\|)$ be an $n$-normed space of dimension $d \geq n \geq 2$ and $\left\{a_{1}, a_{2}, \ldots, a_{n}\right\}$ be linearly independent set in $X$. Then the following function $\|\cdot, \ldots, \cdot\|_{\infty}$ on $X^{n-1}$ defined by

$$
\begin{aligned}
& \left\|x_{1}, x_{2}, \ldots, x_{n}\right\|_{\infty} \\
& \quad=\max \left\{\left\|x_{1}, x_{2}, \ldots, x_{n-1}, a_{i}\right\|: i=1,2, \ldots, n\right\}
\end{aligned}
$$

defines an $(n-1)$-norm on $X$ with respect to $\left\{a_{1}, a_{2}, \ldots, a_{n}\right\}$.

A sequence $\left(x_{k}\right)$ in an $n$-normed space $(X,\|\cdot, \ldots, \cdot\|)$ is said to converge to some $L \in X$ if

$\lim _{k \rightarrow \infty}\left\|x_{k}-L, z_{1}, \ldots, z_{n-1}\right\|=0 \quad$ for every $z_{1}, \ldots, z_{n-1} \in X$

A sequence $\left(x_{k}\right)$ in an $n$-normed space $(X,\|\cdot, \ldots, \cdot\|)$ is said to be Cauchy if

$$
\begin{array}{r}
\lim _{\substack{k \rightarrow \infty \\
p \rightarrow \infty}}\left\|x_{k}-x_{p}, z_{1}, \ldots, z_{n-1}\right\|=0 \\
\text { for every } z_{1}, \ldots, z_{n-1} \in X .
\end{array}
$$

If every Cauchy sequence in $X$ converges to some $L \in X$, then $X$ is said to be complete with respect to the $n$-norm. Any complete $n$-normed space is said to be $n$-Banach space.

An Orlicz function $M$ is a function which is continuous, nondecreasing, and convex with $M(0)=0, M(x)>0$ for $x>0$ and $M(x) \rightarrow \infty$ as $x \rightarrow \infty$. 
Lindenstrauss and Tzafriri [6] used the idea of Orlicz function to define the following sequence space. Let $w$ be the space of all real or complex sequences $x=\left(x_{k}\right)$; then

$$
\ell_{M}=\left\{x \in w: \sum_{k=1}^{\infty} M\left(\frac{\left|x_{k}\right|}{\rho}\right)<\infty\right\}
$$

which is called an Orlicz sequence space. The space $\ell_{M}$ is a Banach space with the norm

$$
\|x\|=\inf \left\{\rho>0: \sum_{k=1}^{\infty} M\left(\frac{\left|x_{k}\right|}{\rho}\right) \leq 1\right\} .
$$

It is shown in [6] that every Orlicz sequence space $\ell_{M}$ contains a subspace isomorphic to $\ell_{p}(p \geq 1)$. The $\Delta_{2^{-}}$ condition is equivalent to $M(L x) \leq k L M(x)$ for all values of $x \geq 0$ and for $L>1$. A sequence $\mathscr{M}=\left(M_{k}\right)$ of Orlicz function is called a Musielak-Orlicz function; see $[7,8]$. A sequence $\mathcal{N}=\left(N_{k}\right)$ defined by

$$
N_{k}(v)=\sup \left\{|v| u-\left(M_{k}\right): u \geq 0\right\}, \quad k=1,2, \ldots,
$$

is called the complementary function of a Musielak-Orlicz function $\mathscr{M}$. For a given Musielak-Orlicz function $\mathscr{M}$, the Musielak-Orlicz sequence space $t_{\mathscr{M}}$ and its subspace $h_{\mathscr{M}}$ are defined as follows:

$$
\begin{gathered}
t_{\mathscr{M}}=\left\{x \in w: I_{\mathscr{M}}(c x)<\infty \text { for some } c>0\right\}, \\
h_{\mathscr{M}}=\left\{x \in w: I_{\mathscr{M}}(c x)<\infty \forall c>0\right\},
\end{gathered}
$$

where $I_{\mathscr{M}}$ is a convex modular defined by

$$
I_{\mathscr{M}}(x)=\sum_{k=1}^{\infty} M_{k}\left(x_{k}\right), \quad x=\left(x_{k}\right) \in t_{\mathscr{M}} .
$$

We consider $t_{\mathscr{M}}$ equipped with the Luxemburg norm

$$
\|x\|=\inf \left\{k>0: I_{\mathscr{M}}\left(\frac{x}{k}\right) \leq 1\right\}
$$

or equipped with the Orlicz norm

$$
\|x\|^{0}=\inf \left\{\frac{1}{k}\left(1+I_{\mathscr{M}}(k x)\right): k>0\right\} .
$$

Let $X$ be a linear metric space. A function $p: X \rightarrow \mathbb{R}$ is called paranorm if

(1) $p(x) \geq 0$ for all $x \in X$,

(2) $p(-x)=p(x)$ for all $x \in X$,

(3) $p(x+y) \leq p(x)+p(y)$ for all $x, y \in X$,

(4) if $\left(\lambda_{n}\right)$ is a sequence of scalars with $\lambda_{n} \rightarrow \lambda$ as $n \rightarrow$ $\infty$, and $\left(x_{n}\right)$ is a sequence of vectors with $p\left(x_{n}-x\right) \rightarrow$ 0 as $n \rightarrow \infty$; then $p\left(\lambda_{n} x_{n}-\lambda x\right) \rightarrow 0$ as $n \rightarrow \infty$.

A paranorm $p$ for which $p(x)=0$ implies $x=0$ is called total paranorm, and the pair $(X, p)$ is called a total paranormed space. It is well known that the metric of any linear metric space is given by some total paranorm (see [ 9 ,
Theorem 10.4.2, p. 183]). For more details about sequence spaces, see [10-23] and the references therein.

Let $\sum_{k=0}^{\infty} a_{k}$ be an infinite series with sequence of partial sums $\left(s_{k}\right)$ and $q$ be any positive real number. The Euler transform $(E, q) s$ of the sequence $s=\left(s_{n}\right)$ is defined by

$$
E_{n}^{q}(s)=\frac{1}{(1+q)^{n}} \sum_{v=0}^{n}\left(\begin{array}{l}
n \\
v
\end{array}\right) q^{n-v} s_{v} .
$$

The series $\sum_{n=0}^{\infty} a_{n}$ is said to be summable $(E, q)$ to the number $s$ if

$$
E_{n}^{q}(s)=\frac{1}{(1+q)^{n}} \sum_{v=0}^{n}\left(\begin{array}{l}
n \\
v
\end{array}\right) q^{n-v} s_{v} \longrightarrow s \quad \text { as } n \longrightarrow \infty
$$

and is said to be absolutely summable $(E, q)$ or summable $|E, q|$ if

$$
\sum_{k}\left|E_{k}^{q}(s)-E_{k-1}^{q}(s)\right|<\infty .
$$

Let $x=\left(x_{n}\right)$ be a sequence of scalars; we denote $N_{n}(x)=E_{n}^{q}(x)-E_{n-1}^{q}(x)$, where $E_{n}^{q}(x)$ is defined by (12). After applications of Abel's transform, we have

$$
\begin{aligned}
N_{n}(x)= & -\frac{1}{(1+q)^{n-1}} \sum_{k=0}^{n-2} x_{k+1} A_{k} \\
& +\frac{s_{n-1} A_{n-1}}{(1+q)^{n-1}}+\frac{s_{n}}{(1+q)^{n}}-\frac{q^{n-1}}{(1+q)^{n}} s_{0}
\end{aligned}
$$

where

$$
A_{k}=\sum_{i=0}^{k}\left[\frac{q}{1+q}\left(\begin{array}{c}
n \\
i
\end{array}\right)-\left(\begin{array}{c}
n-1 \\
i
\end{array}\right)\right] q^{n-i-1} .
$$

Note that, for any sequences $x=\left(x_{n}\right), y=\left(y_{n}\right)$ and scalar $\lambda$, we have $N_{n}(x+y)=N_{n}(x)+N_{n}(y)$ and $N_{n}(\lambda x)=$ $\lambda N_{n}(x)$.

Let $\mathscr{M}=\left(M_{k}\right)$ be a Musielak-Orlicz function and $p=$ $\left(p_{k}\right)$ be a bounded sequence of positive real numbers. We define the following sequence space in the present paper:

$$
\begin{aligned}
& E_{n}^{q}(\mathscr{M}, p, s,\|\cdot, \ldots, \cdot\|) \\
& =\left\{x=\left(x_{k}\right): \sum_{k=1}^{\infty} \frac{1}{k^{s}}\left[M_{k}\left(\left\|\frac{N_{k}(x)}{\rho}, z_{1}, \ldots, z_{n-1}\right\|\right)\right]^{p_{k}}<\infty,\right. \\
& \quad s \geq 0, \text { for some } \rho>0\} .
\end{aligned}
$$

If we take $p=\left(p_{k}\right)=1$ for all $k \in \mathbb{N}$, we have

$$
\begin{aligned}
& E_{n}^{q}(\mathscr{M}, s,\|\cdot, \ldots, \cdot\|) \\
& =\left\{x=\left(x_{k}\right): \sum_{k=1}^{\infty} \frac{1}{k^{s}}\left[M_{k}\left(\left\|\frac{N_{k}(x)}{\rho}, z_{1}, \ldots, z_{n-1}\right\|\right)\right]<\infty,\right. \\
& \quad s \geq 0, \text { for some } \rho>0\} .
\end{aligned}
$$


If we take $s=0$, we get

$$
\begin{aligned}
E_{n}^{q}(\mathscr{M}, p,\|\cdot, \ldots, \cdot\|) & \\
= & \left\{x=\left(x_{k}\right): \sum_{k=1}^{\infty}\left[M_{k}\left(\left\|\frac{N_{k}(x)}{\rho}, z_{1}, \ldots, z_{n-1}\right\|\right)\right]^{p_{k}}\right. \\
& <\infty, s \geq 0, \text { for some } \rho>0\} .
\end{aligned}
$$

The following inequality will be used throughout the paper. If $0 \leq p_{k} \leq \sup p_{k}=H, K=\max \left(1,2^{H-1}\right)$, then

$$
\left|a_{k}+b_{k}\right|^{p_{k}} \leq K\left\{\left|a_{k}\right|^{p_{k}}+\left|b_{k}\right|^{p_{k}}\right\}
$$

for all $k$ and $a_{k}, b_{k} \in \mathbb{C}$. Also, $|a|^{p_{k}} \leq \max \left(1,|a|^{H}\right)$ for all $a \in \mathbb{C}$.

In this paper, we study some topological properties and inclusion relations between the previously defined sequence spaces.

\section{Main Results}

Theorem 1. Let $\mathscr{M}=\left(M_{k}\right)$ be a Musielak-Orlicz function and $p=\left(p_{k}\right)$ be a bounded sequence of positive real numbers; the space $E_{n}^{q}(\mathscr{M}, p, s,\|\cdot, \ldots, \cdot\|)$ is linear over the complex field $\mathbb{C}$.

Proof. Let $x=\left(x_{k}\right), y=y_{k} \in E_{n}^{q}(\mathscr{M}, p, s,\|\cdot, \ldots, \cdot\|)$, and $\alpha, \beta \in \mathbb{C}$. Then, there exist positive integers $\rho_{1}$ and $\rho_{2}$ such that

$$
\begin{aligned}
& \sum_{k=1}^{\infty} \frac{1}{k^{s}}\left[M_{k}\left(\left\|\frac{N_{k}(x)}{\rho_{1}}, z_{1}, \ldots, z_{n-1}\right\|\right)\right]^{p_{k}}<\infty, \\
& \sum_{k=1}^{\infty} \frac{1}{k^{s}}\left[M_{k}\left(\left\|\frac{N_{k}(y)}{\rho_{2}}, z_{1}, \ldots, z_{n-1}\right\|\right)\right]^{p_{k}}<\infty .
\end{aligned}
$$

Let $\rho_{3}=\max \left(2|\alpha| \rho_{1}, 2|\beta| \rho_{2}\right)$. Since $\left(M_{k}\right)$ is nondecreasing, convex function and by using inequality (20), we have

$$
\begin{gathered}
\sum_{k=1}^{\infty} \frac{1}{k^{s}}\left[M_{k}\left(\left\|\frac{N_{k}(\alpha x+\beta y)}{\rho_{3}}, z_{1}, \ldots, z_{n-1}\right\|\right)\right]^{p_{k}} \\
\leq \sum_{k=1}^{\infty} \frac{1}{k^{s}}\left[M_{k}\left(\left\|\frac{N_{k}(\alpha x)}{\rho_{3}}, z_{1}, \ldots, z_{n-1}\right\|\right)\right. \\
\left.\quad+\left(\left\|\frac{N_{k}(\beta y)}{\rho_{3}}, z_{1}, \ldots, z_{n-1}\right\|\right)\right]^{p_{k}} \\
\leq K \sum_{k=1}^{\infty} \frac{1}{k^{s}}\left[M_{k}\left(\left\|\frac{N_{k}(x)}{\rho_{1}}, z_{1}, \ldots, z_{n-1}\right\|\right)\right]^{p_{k}} \\
\quad+K \sum_{k=1}^{\infty} \frac{1}{k^{s}}\left[M_{k}\left(\left\|\frac{N_{k}(y)}{\rho_{2}}, z_{1}, \ldots, z_{n-1}\right\|\right)\right]^{p_{k}} \\
<\infty .
\end{gathered}
$$

Therefore, $\alpha x+\beta y \in E_{n}^{q}(\mathscr{M}, p, s,\|\cdot, \ldots, \cdot\|)$. Hence, $E_{n}^{q}(\mathscr{M}$, $p, s,\|\cdot, \ldots, \cdot\|)$ is a linear space.
Theorem 2. Let $\mathscr{M}=\left(M_{k}\right)$ be a Musielak-Orlicz function and $p=\left(p_{k}\right)$ be a bounded sequence of positive real numbers. Then, the space $E_{n}^{q}(\mathscr{M}, p, s,\|\cdot, \ldots, \cdot\|)$ is a paranormed space with

$$
\begin{aligned}
& g(x) \\
& =\inf \left\{\rho^{p_{n} / H}:\left(\sum_{k=1}^{\infty} \frac{1}{k^{s}}\left[M_{k}\left(\left\|\frac{N_{k}(x)}{\rho}, z_{1}, \ldots, z_{n-1}\right\|\right)\right]^{p_{k}}\right)^{1 / H}\right. \\
& \leq 1, n=1,2,3, \ldots\},
\end{aligned}
$$

where $H=\max \left(1, \sup _{k} p_{k}\right)$.

Proof. Clearly, $g(x)=g(-x)$ and $g(x+y) \leq g(x)+g(y)$. Since $M_{k}(0)=0$, we get $\inf \left\{\rho^{p_{n} / H}\right\}=0$ for $x=0$. Finally, we prove that multiplication is continuous. Let $\lambda$ be any number such that

$$
\begin{aligned}
& g(\lambda x) \\
& =\inf \left\{\rho^{p_{n} / H}: \sum_{k=1}^{\infty} \frac{1}{k^{s}}\left[M_{k}\left(\left\|\frac{\lambda N_{k}(x)}{\rho}, z_{1}, \ldots, z_{n-1}\right\|\right)\right]^{p_{k}}\right. \\
& \leq 1, n=1,2,3, \ldots\} .
\end{aligned}
$$

Thus, we have

$$
\begin{gathered}
g(\lambda x)=\inf \left\{(\lambda s)^{p_{n} / H}: \sum_{k=1}^{\infty} \frac{1}{k^{s}}\left[M_{k}\left(\left\|\frac{N_{k}(x)}{s}, z_{1}, \ldots, z_{n-1}\right\|\right)\right]^{p_{k}}\right. \\
\leq 1, n=1,2,3, \ldots\}
\end{gathered}
$$

where $s=\rho /|\lambda|$. Since $|\lambda|^{p_{k}} \leq \max \left(1,|\lambda|^{H}\right)$, then $|\lambda|^{p_{k} / H} \leq$ $\left(\max \left(1,|\lambda|^{H}\right)\right)^{1 / H}$. Hence,

$g(\lambda x)$

$$
\begin{aligned}
& \leq\left(\max \left(1,|\lambda|^{H}\right)\right)^{1 / H} \\
& \inf \left\{(s)^{p_{n} / H}:\left(\sum_{k=1}^{\infty} \frac{1}{k^{s}}\left[M_{k}\left(\left\|\frac{N_{k}(x)}{\rho}, z_{1}, \ldots, z_{n-1}\right\|\right)\right]^{p_{k}}\right)^{1 / H}\right. \\
& \leq 1, n=1,2, \ldots\},
\end{aligned}
$$

and therefore $g(\lambda x)$ converges to zero when $g(x)$ converges to zero in $E_{n}^{q}(\mathscr{M}, p, s,\|\cdot, \ldots, \cdot\|)$. Now, suppose that $\lambda_{n} \rightarrow 0$ 
as $n \rightarrow \infty$ and $x$ in $E_{n}^{q}(\mathscr{M}, p, s,\|\cdot, \ldots, \cdot\|)$. For arbitrary $\epsilon>0$, let $n_{0}$ be a positive integer such that

$$
\sum_{k=n_{0}+1}^{\infty} \frac{1}{k^{s}}\left[M_{k}\left(\left\|\frac{N_{k}(x)}{\rho}, z_{1}, \ldots, z_{n-1}\right\|\right)\right]^{p_{k}}<\frac{\epsilon}{2}
$$

for some $\rho>0$. This implies that

$$
\left(\sum_{k=n_{0}+1}^{\infty} \frac{1}{k^{s}}\left[M_{k}\left(\left\|\frac{N_{k}(x)}{\rho}, z_{1}, \ldots, z_{n-1}\right\|\right)\right]^{p_{k}}\right)^{1 / H} \leq \frac{\epsilon}{2} .
$$

Let $0<|\lambda|<1$; then, using convexity of $\left(M_{k}\right)$, we get

$$
\begin{aligned}
& \sum_{k=n_{0}+1}^{\infty} \frac{1}{k^{s}}\left[M_{k}\left(\left\|\frac{\lambda N_{k}(x)}{\rho}, z_{1}, \ldots, z_{n-1}\right\|\right)\right]^{p_{k}} \\
& \quad<|\lambda| \sum_{k=n_{0}+1}^{\infty} \frac{1}{k^{s}}\left[M_{k}\left(\left\|\frac{N_{k}(x)}{\rho}, z_{1}, \ldots, z_{n-1}\right\|\right)\right]^{p_{k}} \\
& <\left(\frac{\epsilon}{2}\right)^{H} .
\end{aligned}
$$

Since $\left(M_{k}\right)$ is continuous everywhere in $[0, \infty)$, then

$$
h(t)=\sum_{k=1}^{n_{0}} \frac{1}{k^{s}}\left[M_{k}\left(\left\|\frac{t N_{k}(x)}{\rho}, z_{1}, \ldots, z_{n-1}\right\|\right)\right]^{p_{k}}
$$

is continuous at 0 . So there is $0<\delta<1$ such that $|h(t)|<\epsilon / 2$ for $0<t<\delta$. Let $K$ be such that $\left|\lambda_{n}\right|<\delta$ for $n>K$; we have

$$
\left(\sum_{k=1}^{n_{0}} \frac{1}{k^{s}}\left[M_{k}\left(\left\|\frac{\lambda_{n} N_{k}(x)}{\rho}, z_{1}, \ldots, z_{n-1}\right\|\right)\right]^{p_{k}}\right)^{1 / H}<\frac{\epsilon}{2} .
$$

Thus,

$$
\left(\sum_{k=1}^{\infty} \frac{1}{k^{s}}\left[M_{k}\left(\left\|\frac{\lambda_{n} N_{k}(x)}{\rho}, z_{1}, \ldots, z_{n-1}\right\|\right)\right]^{p_{k}}\right)^{1 / H}<\epsilon
$$

for $n>K$.

Hence, $g(\lambda x) \rightarrow 0$ as $\lambda \rightarrow 0$. This completes the proof of the theorem.

Theorem 3. If $\mathscr{M}^{\prime}=\left(M_{k}^{\prime}\right)$ and $\mathscr{M}^{\prime \prime}=\left(M_{k}^{\prime \prime}\right)$ are two Musielak-Orlicz functions and $s, s_{1}$, and $s_{2}$ are nonnegative real numbers, then one has

(i) $E_{n}^{q}\left(\mathscr{M}^{\prime}, p, s,\|\cdot, \ldots, \cdot\|\right) \cap E_{n}^{q}\left(\mathscr{M}^{\prime \prime}, p, s,\|\cdot, \ldots, \cdot\|\right) \subseteq$ $E_{n}^{q}\left(\mathscr{M}^{\prime}+\mathscr{M}^{\prime \prime}, p, s,\|\cdot, \ldots, \cdot\|\right)$,

(ii) If $s_{1} \leq s_{2}$, then $E_{n}^{q}\left(\mathscr{M}^{\prime}, p, s_{1},\|\cdot, \ldots, \cdot\|\right) \subseteq E_{n}^{q}\left(\mathscr{M}^{\prime}\right.$, $\left.p, s_{2},\|\cdot, \ldots, \cdot\|\right)$

(iii) If $\mathscr{M}^{\prime}$ and $\mathscr{M}^{\prime \prime}$ are equivalent, then $E_{n}^{q}\left(\mathscr{M}^{\prime}, p, s,\|\cdot, \ldots, \cdot\|\right)=E_{n}^{q}\left(\mathscr{M}^{\prime \prime} p, s,\|\cdot, \ldots, \cdot\|\right)$.

Proof. It is obvious, so we omit the details.
Theorem 4. Suppose that $0<r_{k} \leq p_{k}<\infty$ for each $k$. Then $E_{n}^{q}(\mathscr{M}, r, s,\|\cdot \ldots, \cdot\|) \subseteq E_{n}^{q}(\mathscr{M}, p, s,\|\cdot, \ldots, \cdot\|)$.

Proof. Let $x \in E_{n}^{q}(\mathscr{M}, r, s,\|\cdot, \ldots, \cdot\|)$. Then there exists some $\rho>0$ such that

$$
\sum_{k=1}^{\infty} \frac{1}{k^{s}}\left[M_{k}\left(\left\|\frac{N_{k}(x)}{\rho}, z_{1}, \ldots, z_{n-1}\right\|\right)\right]^{r_{k}}<\infty .
$$

This implies that $M_{k}\left(\left\|N_{k}(x) / \rho, z_{1}, \ldots, z_{n-1}\right\|\right) \leq 1$ for sufficiently large value of $k$, say $k \geq k_{0}$, for some fixed $k_{0} \in \mathbb{N}$. Since $\left(M_{k}\right)$ is nondecreasing, we get

$$
\begin{aligned}
\sum_{k \geq k_{0}}^{\infty} \frac{1}{k^{s}} & {\left[M_{k}\left(\left\|\frac{N_{k}(x)}{\rho}, z_{1}, \ldots, z_{n-1}\right\|\right)\right]^{p_{k}} } \\
& \leq \sum_{k \geq k_{0}}^{\infty} \frac{1}{k^{s}}\left[M_{k}\left(\left\|\frac{N_{k}(x)}{\rho}, z_{1}, \ldots, z_{n-1}\right\|\right)\right]^{r_{k}}<\infty .
\end{aligned}
$$

Hence, $x \in E_{n}^{q}(\mathscr{M}, p, s,\|\cdot, \ldots, \cdot\|)$.

Theorem 5. (i) If $0<p_{k} \leq 1$ for each $k$, then $E_{n}^{q}(\mathscr{M}, p, s,\|\cdot, \ldots, \cdot\|) \subseteq E_{n}^{q}(\mathscr{M}, s,\|\cdot, \ldots, \cdot\|)$.

(ii) If $p_{k} \geq 1$ for all $k$, then $E_{n}^{q}(\mathscr{M}, s,\|\cdot, \ldots, \cdot\|) \subseteq$ $E_{n}^{q}(\mathscr{M}, p, s,\|\cdot, \ldots, \cdot\|)$.

Proof. It is easy to prove by using Theorem 4; so we omit the details.

\section{Statistical Convergence}

The notion of statistical convergence was introduced by Fast [24] and Schoenberg [25] independently. Over the years and under different names, statistical convergence has been discussed in the theory of Fourier analysis, ergodic theory, and number theory. Later on, it was further investigated from the sequence space point of view and linked with summability theory by Fridy [26], Connor [27], Šalát [28], Mursaleen [29], Işik [30], Savaş [31], Malkowsky and Savas [32], Kolk [33], Maddox [34], Mohiuddine and Aiyub [35], and many others. In the recent years, generalizations of statistical convergence have appeared in the study of strong integral summability and the structure of ideals of bounded continuous functions on locally compact spaces. Statistical convergence and its generalizations are also connected with subsets of the Stone-Cech compactification of natural numbers. Moreover, statistical convergence is closely related to the concept of convergence in probability.

Let $\theta=\left(k_{r}\right)$ be the sequence of positive integers such that $k_{0}=0,0<k_{r}<k_{r+1}$ and $h_{r}=k_{r}-k_{r-1} \rightarrow \infty$ as $r \rightarrow \infty$. Then $\theta$ is called a lacunary sequence. The intervals determined by $\theta$ will be denoted by $I_{r}=\left(k_{r-1}, k_{r}\right]$, and the ratio $k_{r} /\left(k_{r}-1\right)$ will be denoted by $q_{r}$ (see [36]).

Let $\theta=\left(k_{r}\right)$ be a lacunary sequence; then the sequence $x=\left(x_{k}\right)$ is said to be $N_{k}(u)$-lacunary statistically convergent to the number $l$ provided that, for every $\epsilon>0$,

$$
\lim _{r} \frac{1}{h_{r}}\left|\left\{k \in I_{r}:\left\|\frac{u_{k} N_{k}(x)-l}{\rho}, z_{1}, \ldots, z_{n-1}\right\| \geq \epsilon\right\}\right|=0 .
$$


In this case, we write $\left[N_{k}(u), S\right]_{\theta}-\lim x=l$ or $x_{k} \rightarrow$ $l\left(\left[N_{k}(u), S\right]_{\theta}\right)$.

Let $\theta=\left(k_{r}\right)$ be a lacunary sequence, $\mathscr{M}=\left(M_{k}\right)$ be a Musielak-Orlicz function, $u=\left(u_{k}\right)$ be a sequence of strictly positive real numbers, and $p=\left(p_{k}\right)$ be a bounded sequence of positive real numbers. A sequence $x=\left(x_{k}\right)$ is said to be strongly $N_{k}(u)$-lacunary convergent to the number $l$ with respect to the Musielak-Orlicz function $\mathscr{M}$ provided that

$$
\lim _{r} \frac{1}{h_{r}} \sum_{k \in I_{r}}\left[M_{k}\left(\left\|\frac{u_{k} N_{k}(x)-l}{\rho}, z_{1}, \ldots, z_{n-1}\right\|\right)\right]^{p_{k}}=0 .
$$

The set of all strongly $N_{k}(u)$-lacunary convergent sequences to the number $l$ with respect to the MusielakOrlicz function $\mathscr{M}$ is denoted by $\left[N_{k}, u, \mathscr{M}, p,\|\cdot, \ldots, \cdot\|\right]_{\theta}$. In this case, we write $x_{k} \rightarrow l\left(\left[N_{k}, u, \mathscr{M}, p,\|\cdot, \ldots, \cdot\|\right]_{\theta}\right)$. In the special case $\mathscr{M}(x)=x, p_{k}=p_{0}$ for all $k \in \mathbb{N}$, we shall write $\left[N_{k}, u,\|\cdot, \ldots, \cdot\|\right]_{\theta}$ instead of $\left[N_{k}, u, \mathscr{M}, p,\|\cdot, \ldots, \cdot\|\right]_{\theta}$.

In this section, we give some results about $N_{k}(u)$-lacunary statistical convergence and give some relations between the set of $N_{k}(u)$-lacunary statistical convergence sequences and other spaces which are defined with respect to MusielakOrlicz function $\mathscr{M}$.

Theorem 6. Let $\theta=\left(k_{r}\right)$ be a lacunary sequence.

(i) If a sequence $x=\left(x_{k}\right)$ is strongly $N_{k}(u)$-lacunary convergent to $l$, then it is $N_{k}(u)$-lacunary statistically convergent to $l$.

(ii) If a bounded sequence $x=\left(x_{k}\right)$ is $N_{k}(u)$-lacunary statistically convergent to $l$, then it is strongly $N_{k}(u)$ lacunary convergent to $l$.

Proof. (i) Let $\epsilon>0$ and $x_{k} \rightarrow l\left(\left[N_{k}, u,\|\cdot, \ldots, \cdot\|\right]_{\theta}\right)$. Then, we can write

$$
\begin{aligned}
& \sum_{k \in I_{r}}\left\|\frac{u_{k} N_{k}(x)-l}{\rho}, z_{1}, \ldots, z_{n-1}\right\|^{p_{0}} \\
& \quad \geq \sum_{\substack{k \in I_{r}\\
}}\left\|\frac{u_{k} N_{k}(x)-l}{\rho}, z_{1}, \ldots, z_{n-1}\right\|^{p_{0}} \\
& \quad \geq \epsilon^{p_{0}}\left|\left\{k \in I_{r}:\left\|\frac{\left.u_{k} N_{k}(x)-l\right) / \rho, z_{1}, \ldots, z_{n-1} \| \geq \epsilon}{\rho}, z_{1}, \ldots, z_{n-1}\right\| \geq \epsilon\right\}\right| .
\end{aligned}
$$

Hence, $x_{k} \rightarrow l\left(\left[N_{k}, u, S\right]_{\theta}\right)$.

(ii) Suppose that $x_{k} \rightarrow l\left(\left[N_{k}, u, S\right]_{\theta}\right)$, and let $x \in l_{\infty}$. Let $\epsilon>0$ be given and take $N_{\epsilon}$ such that

$$
\begin{aligned}
& \frac{1}{h_{r}}\left|\left\{k \in I_{r}:\left\|\frac{u_{k} N_{k}(x)-l}{\rho}, z_{1}, \ldots, z_{n-1}\right\| \geq\left(\frac{\epsilon}{2}\right)^{\left(1 / p_{0}\right)}\right\}\right| \\
& \leq \frac{\epsilon}{2 K^{p_{0}}}
\end{aligned}
$$

for all $r>N_{\epsilon}$ and set $T_{r}=\left\{k \in I_{r}: \|\left(u_{k} N_{k}(x)-\right.\right.$ l) $\left./ \rho, z_{1}, \ldots, z_{n-1} \| \geq(\epsilon / 2)^{1 / p_{0}}\right\}$, where $K=\sup _{k}\left|x_{k}\right|<\infty$. Now, for all $r>N_{\epsilon}$, we have

$$
\begin{aligned}
& \frac{1}{h_{r}} \sum_{k \in I_{r}}\left\|\frac{u_{k} N_{k}(x)-l}{\rho}, z_{1}, \ldots, z_{n-1}\right\|^{p_{0}} \\
& =\frac{1}{h_{r}} \sum_{\substack{k \in I_{r} \\
k \in T_{r}}}\left\|\frac{u_{k} N_{k}(x)-l}{\rho}, z_{1}, \ldots, z_{n-1}\right\|^{p_{0}} \\
& +\frac{1}{h_{r}} \sum_{\substack{k \in I_{r} \\
k \notin T_{r}}}\left\|\frac{u_{k} N_{k}(x)-l}{\rho}, z_{1}, \ldots, z_{n-1}\right\|^{p_{0}} \\
& \leq \frac{1}{h_{r}}\left(\frac{h_{r} \epsilon}{2 K^{p_{0}}}\right) K^{p_{0}}+\frac{\epsilon}{2 h_{r}} h_{r}=\epsilon .
\end{aligned}
$$

Thus, $\left(x_{k}\right) \in\left[N_{k}, u,\|\cdot, \ldots, \cdot\|\right]_{\theta}$. This completes the proof of the theorem.

Theorem 7. For any lacunary sequence $\theta$, if $\lim _{r \rightarrow \infty}$ inf $q_{r}>$ 1 , then $\left[N_{k}, u, S\right] \subset\left[N_{k}, u, S\right]_{\theta}$.

Proof. If $\lim _{r \rightarrow \infty}$ inf $q_{r}>1$, then there exists a $\delta>0$ such that $1+\delta \leq q_{r}$ for sufficiently large $r$. Since $h_{r}=k_{r}-k_{r-1}$, we have $k_{r} / h_{r} \leq(1+\delta) / \delta$. Let $x_{k} \rightarrow l\left(\left[N_{k}, u, S\right]\right)$. Then, for every $\epsilon>0$, we have

$$
\begin{aligned}
& \frac{1}{k_{r}}\left|\left\{k \leq k_{r}:\left\|\frac{u_{k} N_{k}(x)-l}{\rho}, z_{1}, \ldots, z_{n-1}\right\| \geq \epsilon\right\}\right| \\
& \quad \geq \frac{1}{k_{r}}\left|\left\{k \in I_{r}:\left\|\frac{u_{k} N_{k}(x)-l}{\rho}, z_{1}, \ldots, z_{n-1}\right\| \geq \epsilon\right\}\right| \\
& \quad \geq \frac{\delta}{1+\delta} \frac{1}{k_{r}}\left|\left\{k \in I_{r}:\left\|\frac{u_{k} N_{k}(x)-l}{\rho}, z_{1}, \ldots, z_{n-1}\right\| \geq \epsilon\right\}\right| .
\end{aligned}
$$

Hence, $\left[N_{k}, u, S\right] \subset\left[N_{k}, u, S\right]_{\theta}$.

Theorem 8. Let $\theta=\left(k_{r}\right)$ be a lacunary sequence, $\mathscr{M}=\left(M_{k}\right)$ be a Musielak-Orlicz function, and $0<h=\inf _{k} p_{k} \leq p_{k} \leq$ $\sup _{k} p_{k}=H$. Then, $\left[N_{k}, u, \mathscr{M},\|\cdot, \ldots, \cdot\|\right]_{\theta} \subset\left[N_{k}, u, S\right]_{\theta}$.

Proof. Let $x \in\left[N_{k}, u, \mathscr{M},\|\cdot, \ldots, \cdot\|\right]_{\theta}$. Then there exists a number $\rho>0$ such that

$$
\frac{1}{h_{r}} \sum_{k \in I_{r}}\left[M_{k}\left(\left\|\frac{u_{k} N_{k}(x)-l}{\rho}, z_{1}, \ldots, z_{n-1}\right\|\right)\right]^{p_{k}} \longrightarrow 0
$$


Then, given $\epsilon>0$, we have

$$
\begin{aligned}
& \frac{1}{h_{r}} \sum_{k \in I_{r}}\left[M_{k}\left(\left\|\frac{u_{k} N_{k}(x)-l}{\rho}, z_{1}, \ldots, z_{n-1}\right\|\right)\right]^{p_{k}} \\
& \geq \frac{1}{h_{r}} \sum_{\left\|\left(u_{k} N_{k}(x)-l\right) / \rho, z_{1}, \ldots, z_{n-1}\right\| \geq \epsilon}\left[M _ { k } \left(\| \frac{u_{k} N_{k}(x)-l}{\rho},\right.\right. \\
& \left.\left.\geq \frac{1}{h_{r}} \quad \sum_{k \in I_{r}} z_{1}, \ldots, z_{n-1} \|\right)\right]^{p_{k}} \\
& \geq \frac{1}{h_{r}} \sum \min \left\{\left[M_{k}\left(\epsilon_{1}\right)\right]^{p_{k}},\right. \\
& \geq \frac{1}{h_{r}}\left|\left\{k \in I_{r}:\left\|\frac{\left.\left.u_{k} N_{k}(x)\right]^{h},\left[M_{k}\left(\epsilon_{1}\right)\right]^{H}\right\}}{\rho}, z_{1}, \ldots, z_{n-1}\right\| \geq \epsilon\right\}\right| \\
& \quad \cdot \min \left\{\left[M_{k}\left(\epsilon_{1}\right)\right]^{h},\left[M_{k}\left(\epsilon_{1}\right)\right]^{H}\right\} .
\end{aligned}
$$

Hence, $x \in\left[N_{k}, u, S\right]_{\theta}$. This completes the proof of the theorem.

Theorem 9. Let $\theta=\left(k_{r}\right)$ be a lacunary sequence, $\mathscr{M}=\left(M_{k}\right)$ be a Musielak-Orlicz function, and $p=\left(p_{k}\right)$ be a bounded sequence; then $\left[N_{k}, u, S\right]_{\theta} \subset\left[N_{k}, u, \mathscr{M},\|\cdot \ldots, \cdot\|\right]_{\theta}$.

Proof. Let $x=\left(x_{k}\right) \in l_{\infty}$ with $x_{k} \rightarrow l\left(\left[N_{k}, u, S\right]_{\theta}\right)$. Since $x \in l_{\infty}$, there is a constant $T>0$ such that $\|\left(u_{k} N_{k}(x)-\right.$ $l) / \rho, z_{1}, \ldots, z_{n-1} \| \leq T$, and given $\epsilon>0$, we have

$$
\begin{aligned}
& \frac{1}{h_{r}} \sum_{k \in I_{r}}\left[M_{k}\left(\left\|\frac{u_{k} N_{k}(x)-l}{\rho}, z_{1}, \ldots, z_{n-1}\right\|\right)\right]^{p_{k}} \\
& =\frac{1}{h_{r}} \sum_{\substack{k \in I_{r} \\
\left\|\left(u_{k} N_{k}(x)-l\right) / \rho, z_{1}, \cdots, z_{n-1}\right\| \geq \epsilon}}\left[M _ { k } \left(\| \frac{u_{k} N_{k}(x)-l}{\rho},\right.\right. \\
& \left.\left.z_{1}, \ldots, z_{n-1} \|\right)\right]^{p_{k}} \\
& +\frac{1}{h_{r}} \sum_{\substack{k \in I_{r} \\
\left\|\left(u_{k} N_{k}(x)-l\right) / \rho, z_{1}, \ldots, z_{n-1}\right\|<\epsilon}}\left[M _ { k } \left(\| \frac{u_{k} N_{k}(x)-l}{\rho},\right.\right. \\
& \left.\left.z_{1}, \ldots, z_{n-1} \|\right)\right]^{p_{k}} \\
& \leq \frac{1}{h_{r}} \sum_{\substack{k \in I_{r} \\
\left\|\left(u_{k} N_{k}(x)-l\right) / \rho, z_{1}, \ldots, z_{n-1}\right\| \geq \epsilon}} \max \left\{\left[M_{k}\left(\frac{T}{\rho}\right)\right]^{h},\right. \\
& \left.\left[M_{k}\left(\frac{T}{\rho}\right)\right]^{H}\right\} \\
& +\frac{1}{h_{r}} \sum_{\left\|\left(u_{k} N_{k}(x)-l / \rho\right), z_{1}, \ldots, z_{n-1}\right\|<\epsilon}\left[M_{k}\left(\frac{\epsilon}{\rho}\right)\right]^{p_{k}}
\end{aligned}
$$

$$
\begin{gathered}
\leq \max \left\{\left[M_{k}(K)\right]^{h},\left[M_{k}(K)\right]^{H}\right\} \\
\times \frac{1}{h_{r}}\left|\left\{k \in I_{r}:\left\|\frac{u_{k} N_{k}(x)-l}{\rho}, z_{1}, \ldots, z_{n-1}\right\| \geq \epsilon\right\}\right| \\
+\max \left\{\left[M_{k}\left(\epsilon_{1}\right)\right]^{h},\left[M_{k}\left(\epsilon_{1}\right)\right]^{H}\right\}, \\
\frac{T}{\rho}=K, \quad \frac{\epsilon}{\rho}=\epsilon_{1} .
\end{gathered}
$$

Hence, $x \in\left[N_{k}, u, \mathscr{M},\|\cdot, \ldots, \cdot\|\right]_{\theta}$.

\section{Acknowledgment}

The authors thank the referees for their valuable comments and suggestions.

\section{References}

[1] S. Gähler, "Lineare 2-normierte Räume," Mathematische Nachrichten, vol. 28, pp. 1-43, 1965.

[2] A. Misiak, " $n$-inner product spaces," Mathematische Nachrichten, vol. 140, pp. 299-319, 1989.

[3] H. Gunawan, "On $n$-inner products, $n$-norms, and the CauchySchwarz inequality," Scientiae Mathematicae Japonicae, vol. 5, pp. 47-54, 2001.

[4] H. Gunawan, "The space of $p$-summable sequences and its natural n-norm," Bulletin of the Australian Mathematical Society, vol. 64, no. 1, pp. 137-147, 2001.

[5] H. Gunawan and M. Mashadi, "On n-normed spaces," International Journal of Mathematics and Mathematical Sciences, vol. 27, no. 10, pp. 631-639, 2001.

[6] J. Lindenstrauss and L. Tzafriri, "On Orlicz sequence spaces," Israel Journal of Mathematics, vol. 10, pp. 379-390, 1971.

[7] L. Maligranda, Orlicz Spaces and Interpolation, vol. 5 of Seminários de Matemática, Polish Academy of Science, Warszawa, Poland, 1989.

[8] J. Musielak, Orlicz Spaces and Modular Spaces, vol. 1034 of Lecture Notes in Mathematics, Springer, Berlin, Germany, 1983.

[9] A. Wilansky, Summability through Functional Analysis, vol. 85 of North-Holland Mathematics Studies, North-Holland, Amsterdam, The Netherland, 1984.

[10] F. Basar, Summability Theory and Its Applications, Monographs, Bentham Science Publishers, E-Books, Istanbul, Turkey, 2012.

[11] F. Başar, B. Altay, and M. Mursaleen, "Some generalizations of the space $b v(p)$ of $p$-bounded variation sequences," Nonlinear Analysis: Theory, Methods and Applications A, vol. 68, no. 2, pp. 273-287, 2008.

[12] C. Belen and S. A. Mohiuddine, "Generalized weighted statistical convergence and application," Applied Mathematics and Computation, vol. 219, no. 18, pp. 9821-9826, 2013.

[13] T. Bilgin, "Some new difference sequences spaces defined by an Orlicz function," Filomat, vol. 17, pp. 1-8, 2003.

[14] N. L. Braha and M. Et, "The sequence space $E_{n}^{q}(M, p, s)$ and $N_{k}$-lacunary statistical convergence," Banach Journal of Mathematical Analysis, vol. 7, no. 1, pp. 88-96, 2013.

[15] R. Çolak, B. C. Tripathy, and M. Et, "Lacunary strongly summable sequences and $q$-lacunary almost statistical convergence," Vietnam Journal of Mathematics, vol. 34, no. 2, pp. 129$138,2006$. 
[16] A. M. Jarrah and E. Malkowsky, “The space $b v(p)$, its $\beta$-dual and matrix transformations," Collectanea Mathematica, vol. 55, no. 2, pp. 151-162, 2004.

[17] I. J. Maddox, "Statistical convergence in a locally convex space," Mathematical Proceedings of the Cambridge Philosophical Society, vol. 104, no. 1, pp. 141-145, 1988.

[18] M. Mursaleen, "Generalized spaces of difference sequences," Journal of Mathematical Analysis and Applications, vol. 203, no. 3, pp. 738-745, 1996.

[19] M. Mursaleen, "Matrix transformations between some new sequence spaces," Houston Journal of Mathematics, vol. 9, no. 4, pp. 505-509, 1983.

[20] M. Mursaleen, "On some new invariant matrix methods of summability," The Quarterly Journal of Mathematics, vol. 34, no. 133, pp. 77-86, 1983.

[21] K. Raj and S. K. Sharma, "Some sequence spaces in 2-normed spaces defined by Musielak-Orlicz function," Acta Universitatis Sapientiae. Mathematica, vol. 3, no. 1, pp. 97-109, 2011.

[22] K. Raj and S. K. Sharma, "Some generalized difference double sequence spaces defined by a sequence of Orlicz-functions," Cubo, vol. 14, no. 3, pp. 167-189, 2012.

[23] K. Raj and S. K. Sharma, "Some multiplier sequence spaces defined by a Musielak-Orlicz function in $n$-normed spaces," New Zealand Journal of Mathematics, vol. 42, pp. 45-56, 2012.

[24] H. Fast, "Sur la convergence statistique," Colloquium Mathematicum, vol. 2, pp. 241-244, 1951.

[25] I. J. Schoenberg, "The integrability of certain functions and related summability methods," The American Mathematical Monthly, vol. 66, pp. 361-375, 1959.

[26] J. A. Fridy, “On statistical convergence," Analysis, vol. 5, no. 4, pp. 301-303, 1985.

[27] J. Connor, "A topological and functional analytic approach to statistical convergence," in Analysis of Divergence, Applied and Numerical Harmonic Analysis, pp. 403-413, Birkhäuser, Boston, Mass, USA, 1999.

[28] T. Šalát, "On statistically convergent sequences of real numbers," Mathematica Slovaca, vol. 30, no. 2, pp. 139-150, 1980.

[29] M. Mursaleen, “ $\lambda$-statistical convergence," Mathematica Slovaca, vol. 50, no. 1, pp. 111-115, 2000.

[30] M. Işik, "On statistical convergence of generalized difference sequences," Soochow Journal of Mathematics, vol. 30, no. 2, pp. 197-205, 2004.

[31] E. Savaş, "Strong almost convergence and almost $\lambda$-statistical convergence," Hokkaido Mathematical Journal, vol. 29, no. 3, pp. 531-566, 2000.

[32] E. Malkowsky and E. Savas, "Some $\lambda$-sequence spaces defined by a modulus," Archivum Mathematicum, vol. 36, no. 3, pp. 219$228,2000$.

[33] E. Kolk, "The statistical convergence in Banach spaces," Acta et Commentationes Universitatis Tartuensis, vol. 928, pp. 41-52, 1991.

[34] I. J. Maddox, "A new type of convergence," Mathematical Proceedings of the Cambridge Philosophical Society, vol. 83, no. 1, pp. 61-64, 1978.

[35] S. A. Mohiuddine and M. Aiyub, "Lacunary statistical convergence in random 2-normed spaces," Applied Mathematics \& Information Sciences, vol. 6, no. 3, pp. 581-585, 2012.

[36] A. R. Freedman, J. J. Sember, and M. Raphael, "Some Cesàrotype summability spaces," Proceedings of the London Mathematical Society, vol. 37, no. 3, pp. 508-520, 1978. 


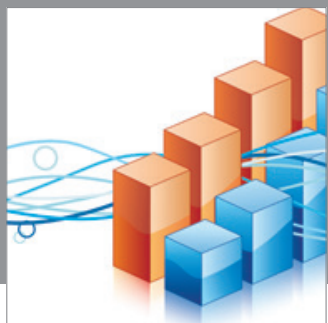

Advances in

Operations Research

mansans

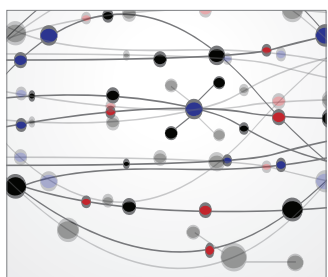

The Scientific World Journal
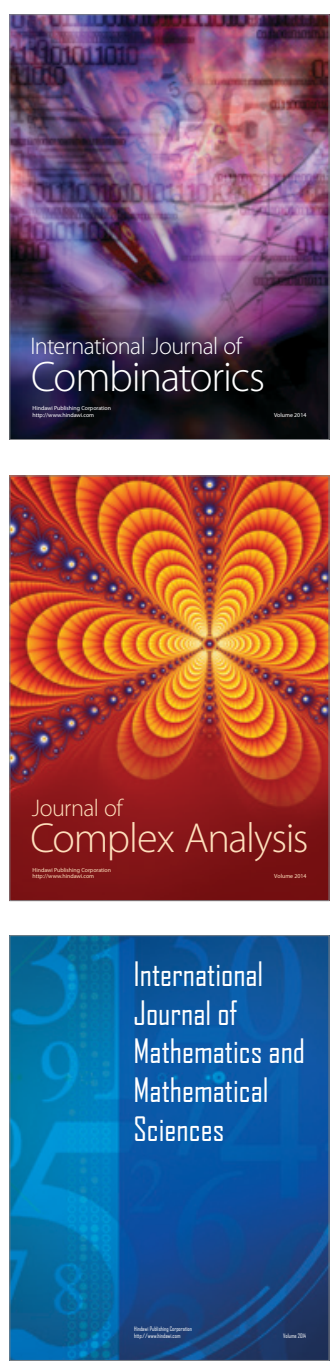
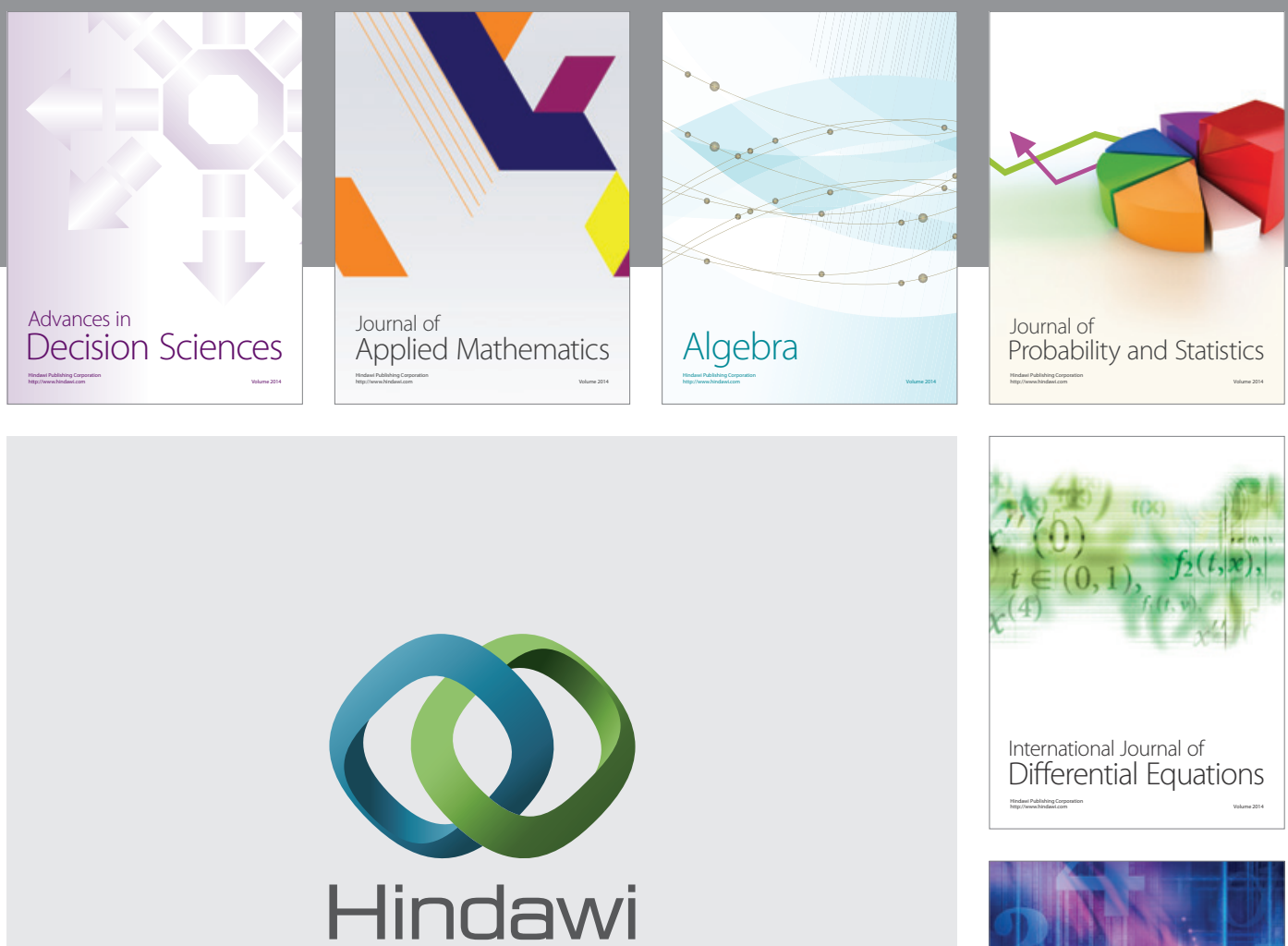

Submit your manuscripts at http://www.hindawi.com
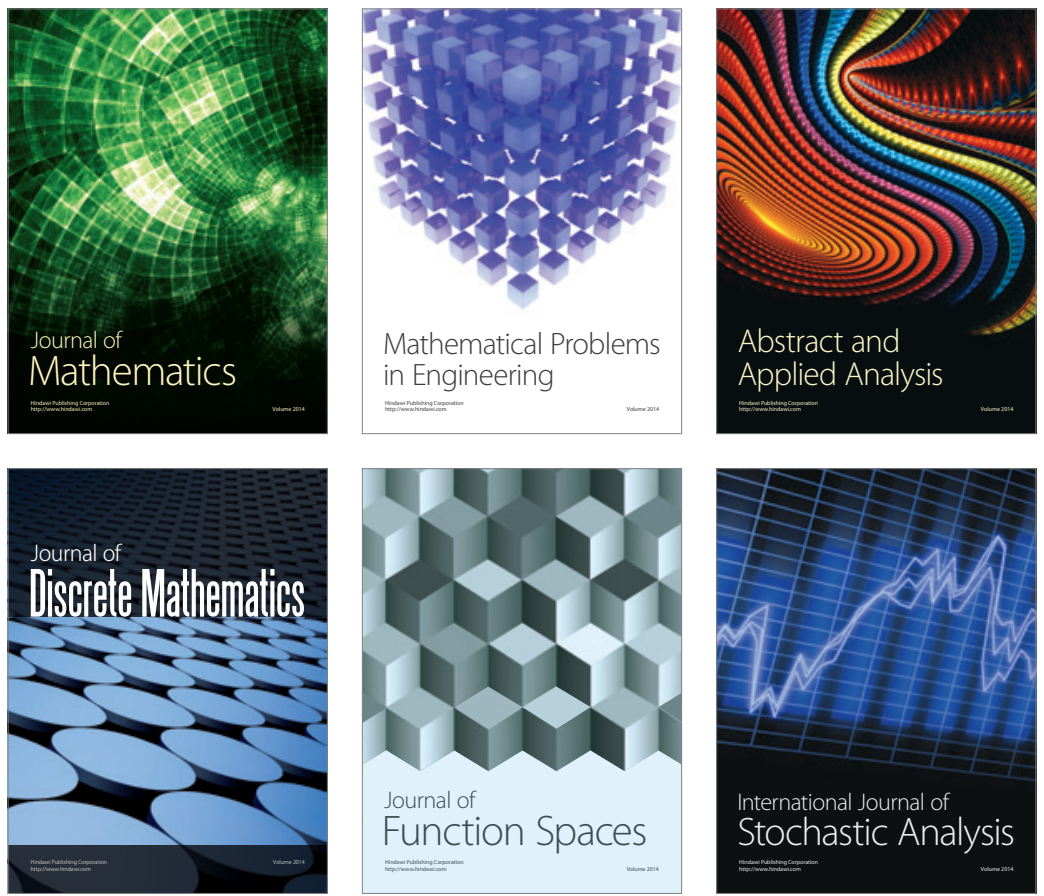

Journal of

Function Spaces

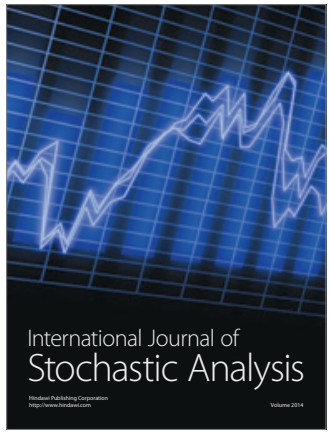

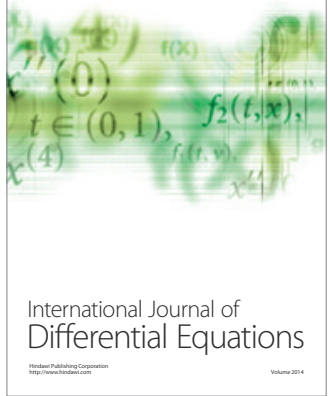
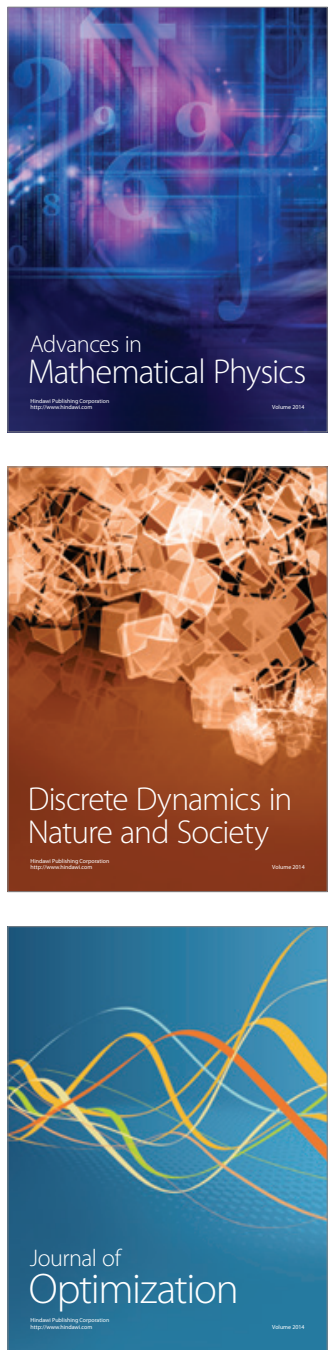